\title{
Review Article \\ Noninvasive Physiologic Assessment of Coronary Stenoses Using Cardiac CT
}

\author{
Lei Xu, ${ }^{1}$ Zhonghua Sun, ${ }^{2}$ and Zhanming Fan ${ }^{1}$ \\ ${ }^{1}$ Department of Radiology, Beijing Anzhen Hospital, Capital Medical University, Beijing 100029, China \\ ${ }^{2}$ Discipline of Medical Imaging, Department of Imaging and Applied Physics, Curtin University, Perth, WA 6845, Australia \\ Correspondence should be addressed to Zhanming Fan; fanzm120@126.com
}

Received 21 June 2014; Revised 29 September 2014; Accepted 6 October 2014

Academic Editor: Michael Ralph Rees

Copyright (c) 2015 Lei Xu et al. This is an open access article distributed under the Creative Commons Attribution License, which permits unrestricted use, distribution, and reproduction in any medium, provided the original work is properly cited.

Coronary CT angiography (CCTA) has become an important noninvasive imaging modality in the diagnosis of coronary artery disease (CAD). CCTA enables accurate evaluation of coronary artery stenosis. However, CCTA provides limited information on the physiological significance of stenotic lesions. A noninvasive "one-stop-shop" diagnostic test that can provide both anatomical significance and functional significance of stenotic lesions would be beneficial in the diagnosis and management of CAD. Recently, with the introduction of novel techniques, such as myocardial CT perfusion, CT-derived fractional flow reserve $\left(\mathrm{FFR}_{\mathrm{CT}}\right)$, and transluminal attenuation gradient (TAG), CCTA has emerged as a noninvasive method for the assessment of both anatomy of coronary lesions and its physiological consequences during a single study. This review provides an overview of the current status of new CT techniques for the physiologic assessments of CAD.

\section{Introduction}

Coronary CT angiography (CCTA) has been widely used as an effective noninvasive imaging modality in the diagnosis of coronary artery disease (CAD) [1]. Multiple-center studies have shown that CCTA allows reliable detection of coronary artery stenoses with high sensitivity and specificity as compared to conventional coronary angiography $[2,3]$. Due to the remarkably high negative predictive value and noninvasive nature, the main strength of CCTA is its excellent ability to exclude significant $\mathrm{CAD}$ in selected patients with intermediate pretest probability $[1,4,5]$. Therefore, CCTA is now well established as an effective "gatekeeper" to invasive coronary angiography and may reduce the rate of normal coronary angiography and improve cost efficiency [6]. Noninvasive assessment of coronary plaque is important for coronary risk stratification. In addition to accurate evaluation of luminal narrowing, CCTA is now a promising approach for noninvasive detection of coronary plaque characteristics, including plaque composition and morphology, and it may therefore contribute to coronary risk stratification [7-9]. A previous study has shown that CCTA-based plaque characterization provides valuable information for the prediction of major cardiovascular events [10]. CCTA not only demonstrates high diagnostic accuracy, but also shows high prognostic value in CAD with very low rate of adverse cardiac events occurring in patients with normal CCTA finding and significantly high rate of these events in patients with obstructive CAD [1113]. CCTA is now a mature diagnostic imaging modality that can provide an effective means to safely guide clinical decision making [14]. One of the limitations of CCTA is that the presence of extensively calcified plaque may cause overestimation of the degree of coronary stenosis, thus resulting in low positive predictive value and potentially leading to increased downstream testing [15-17].

Detection of the physiological severity of intermediate coronary lesions has significant implications for the diagnosis, prognosis, and optimal treatment $[18,19]$. It has been shown that only patients with a hemodynamically significant coronary stenosis benefit from revascularization regardless of the severity of underlying stenosis $[20,21]$. However, in its current form, CCTA is limited in the physiological assessment of coronary atherosclerosis and, therefore, it cannot efficiently discriminate hemodynamically from nonhemodynamically significant stenosis [1]. Studies have shown that a $50 \%$ stenosis identified by CCTA is a poor predictor 
of ischemia [22-24]. Hence, on identification of the significance of CCTA findings, patients often require additional functional tests, as the physiological significance of many lesions remains uncertain. Given these considerations, a noninvasive "one-stop-shop" diagnostic test that can provide both anatomical and hemodynamic significance of stenotic lesions would be beneficial in the diagnosis of CAD. Recently, with the introduction of novel techniques, such as myocardial CT perfusion, noninvasive $\mathrm{FFR}_{\mathrm{CT}}$, and transluminal attenuation gradient, CCTA has emerged as a noninvasive method for the assessment of both anatomy of coronary lesions and its physiological consequences during a single study. This review provides an overview of the current status of new CT techniques for the physiologic assessments of CAD.

\section{Myocardial CT Perfusion Imaging}

Nuclear medicine for a long time has played an important role in the noninvasive evaluation of known or suspected coronary artery disease. Over the past decades, myocardial perfusion imaging (MPI) using single photon emission computed tomography (SPECT) or positron emission tomography (PET) has been well established as the reference standard for the diagnosis and decision making in patients with known coronary artery disease $[25,26]$. A meta-analysis demonstrated that the pooled sensitivity and specificity of SPECT-MPI was $77 \%$ and $77 \%$ on a patient level for the evaluation of functional ischemia with fractional flow reserve as the reference standard [27]. However, SPECT is still limited by low spatial resolution and may miss small or subendocardial areas of hypoperfusion. Attenuation artifact has long been recognized as a major factor limiting the specificity of SPECT for the detection of myocardial perfusion defects [28]. Furthermore, SPECT-MPI may miss some patients with balanced three-vessel disease, as the technique relies on identifying relative differences in perfusion between adjacent myocardial territories [29]. Compared with SPECTMPI, PET-MPI provides higher spatial resolution, accurate attenuation correction, and lower radiation doses for the detection of coronary artery disease $[30,31]$. PET perfusion imaging has an overall sensitivity of $92 \%$ and a specificity of $85 \%$ for the detection of coronary artery disease [32]. However, because of cost and logistics, this approach is not widely available and is not accepted as a clinical routine. The use of hybrid imaging techniques, such as SPECTCT or PET-CT has the advantages of both functional and anatomic imaging. However, these modalities still require two separate imaging procedures, resulting in additional cost and radiation exposure $[22,30]$. The advantage of cardiac magnetic resonance imaging (CMR) over myocardial SPECT is high spatial resolution and not using ionizing radiation [33]. A meta-analysis of CMR perfusion study revealed a sensitivity of $91 \%$ and specificity of $81 \%$ for the diagnosis of CAD on a per-patient level [34]. However, CMR examination is time-consuming as it has long procedure times and limited accuracy in quantification. MR coronary angiography has not been widely accepted as a practical diagnostic tool for CAD. Technical advances with improved spatial and temporal resolution and subsequently reduced scan time and radiation exposure have allowed cardiac CT to simultaneously assess both coronary anatomy and myocardial perfusion.

\section{First-Pass Myocardial CT Perfusion}

Myocardial CT perfusion imaging (CTP) can be performed in two ways: first-pass static perfusion imaging and dynamic CTP. First-pass CCTA imaging has the potential to assess the physiologic significance of coronary artery lesions [35]. In this strategy, rest myocardial CTP is obtained from the images usually acquired during a CCTA examination performed in rest condition. And stress CTP is acquired by an additional CCTA study performed under myocardial stress $[36,37]$.

By using resting CCTA images, coronary artery stenosis and myocardial perfusion may be evaluated simultaneously using the same raw data without the need for further scans, or additional radiation and contrast. However, the value of rest myocardial CTP for the evaluation of myocardial ischemia is uncertain. There have been some reports investigating the detection of myocardial ischemia at rest using multidetector CT (MDCT). Iwasaki and Matsumoto [36] studied the incidence of myocardial perfusion defect by 64 MDCT at rest in patients with significant stenosis and the effect of coronary revascularization therapy on myocardial perfusion. The results demonstrated that a significant percentage of patients with significant coronary stenosis showed myocardial perfusion defect by 64-MDCT at rest, and most of these perfusion defects improved after revascularization therapy. The results indicate that CCTA has the potential for the detection of myocardial ischemia at rest. A study by Busch et al. [38] showed that, in comparison to combined SPECT and CCTA, myocardial CTP using resting CCTA images identifies myocardial infarction with high sensitivity (90\%), good specificity (80\%), and high negative predictive value (NPV) (94\%). The presence of hypoperfusion on CTP suggests either myocardial infarction or ischemia with high predictive power (92\%). In contrast with prior studies, Troupis et al. [39] assessed the sensitivity of 320-detector CT for the detection of myocardial density changes based on rest CCTA by comparing patients with severe coronary artery stenoses (>75\%) and patients with no stenosis. Comparison of ischemic myocardial segments with nonischemic segments demonstrated no significant difference in myocardial density, confirming that myocardial ischemia cannot be reliably detected on rest CCTA. Rest myocardial CTP may have a role in the assessment for myocardial infarction [40, 41]. However, performance of only rest myocardial CTP may be insufficient to reliably rule out reversible ischemia [42].

Stress CTP is performed under pharmacological administration of stress agents, such as adenosine, dipyridamole, or regadenoson, similar to nuclear medicine MPI [43]. In the ischemic cascade, stress perfusion abnormalities are more sensitive than wall motion abnormalities [44]. Myocardial CTP protocols typically include a stress and a rest phase acquisition similar to a nuclear myocardial perfusion imaging examination. When the time interval between the 2 scans is short, the myocardium in the second study may be contaminated by previous injection of contrast material which may decrease the sensitivity for detection of ischemic 
myocardium. Delay time of 10-20 minutes should be used between the two CT scans to allow wash out of contrast from the myocardium. During rest phase acquisition, prospectively ECG-gated imaging can be performed with tube current and voltage tailoring to body mass index to reduce radiation dose. Stress phase imaging is performed with pharmacologically induced stress. The most commonly used stress agent in perfusion imaging is vasodilator agent adenosine. Adenosine is continuously infused at a dose of $0.14 \mathrm{mg} / \mathrm{kg}$ per minute over 3-5 minutes. Stress scan acquisition was performed at peak contrast enhancement with a second bolus injection of contrast [37]. Hypoattenuation areas in the myocardium on CTP represent ischemic myocardium [45]. Generally, if hypoattenuation areas are visualized at stress imaging only, this may indicate reversible myocardial ischemia; if hypoattenuation is visible at rest, this is suggestive of myocardial infarction [43].

Studies have been conducted to investigate the diagnostic performance of stress CTP for the evaluation of CAD. Blankstein et al. [46] studied the diagnostic accuracy of adenosine-stress CTP for identification of hemodynamically significant stenosis compared with nuclear MPI using invasive angiography as the reference standard. The stress CTP had $96 \%$ sensitivity, $73 \%$ specificity, and $98 \%$ NPV on pervessel basis for the detection of stenosis $\geq 70 \%$. Adenosine stress CTP has comparable diagnostic accuracy to SPECT in detecting stress-induced myocardial perfusion defects. The comparison of stress myocardial perfusion imaging with invasive fractional flow reserve (FFR) has been performed by Ko et al. [47]. In this study, forty-two patients with significant stenosis on invasive angiography were included with use of 320-detector row CT. The sensitivity, specificity, positive predictive value (PPV), and NPV of CTP on pervessel territory were $76 \%, 84 \%, 82 \%$, and $79 \%$, respectively. Combining a $\geq 50 \%$ stenosis on CCTA and perfusion defect on CTP was $98 \%$ specific for ischemia, while the presence of normal perfusion on CTP and $<50 \%$ stenosis on CCTA was $100 \%$ specific for exclusion of ischemia. Feuchtner et al. [33] reported stress myocardial CTP using dual-source CT (DSCT) high-pitch mode for detecting reversible ischemia with the comparison of MRI. Stress CTP had sensitivity of $96 \%$, specificity of $88 \%$, PPV of $93 \%$, and NPV of $94 \%$ on pervessel basis. The accuracy increased from $84 \%$ to $95 \%$ after adding stress CTP to CCTA. The results suggest that stress myocardial CTP imaging with 128-slice high-pitch DSCT is feasible for accurate detection of reversible myocardial ischemia.

CORE320 is a multicenter multinational diagnostic study which was designed to evaluate the diagnostic performance of 320-MDCT for detecting coronary stenosis and myocardial perfusion deficits in patients with suspected CAD compared with conventional coronary angiography and SPECT MPI [48]. In this study, 381 patients who underwent combined CCTA-CTP and SPECT-MPI prior to conventional coronary angiography were enrolled from sixteen centers [49]. With the advantage of temporal uniformity achieved by the use of wide detector 320-MDCT system, the diagnostic accuracy of combined CCTA and CTP for detecting or excluding flowlimiting CAD was 0.87 on a patient-basis (defined by area under the receiver operator characteristics curve, ROC). The sensitivity, specificity, PPV, and NPV of integrated CCTA stenosis and CTP perfusion deficit were $80 \%, 74 \%, 65 \%$, and $86 \%$, respectively. The accuracy of CCTA was significantly improved by adding CTP at both the patient and vessel levels. The study indicates that the combination of CCTA and CTP correctly identifies patients with flow-limiting CAD. More recently, one study showed that combining CCTA and CTP improves the diagnostic performance of coronary instent restenosis and CAD in patients with stents compared with CCTA alone [50]. The study is meaningful for clinical decision making in these symptomatic patients.

The use of adenosine during stress CTP may increase the heart rate. CTP is prone to motion artifacts particularly in patients with high heart rate, which may compromise the evaluation of myocardial perfusion. Combined CCTA/CTP has been proven to have better diagnostic performance than CCTA alone. Clinical outcome studies are still needed to determine the effectiveness of stress CTP in evaluating patients with known or suspected CAD compared with other modalities.

\section{Dual-Energy Myocardial CT Perfusion}

Recently, CTP has been performed by using the dualenergy mode. Dual-energy CT (DECT) is based on the principle that body tissues and iodinated contrast have specific spectral characteristics at different energy of Xrays $[51,52]$. DECT acquisition can be classified into two categories: source-oriented and detector-oriented. Currently, two popular approaches for performing DECT are either with dual-source system to produce $\mathrm{X}$-rays with two different tube voltages or with a single X-ray tube switching rapidly between low and high kilovoltage during a single scan [53]. After acquisition of the high- and low-energy data, the iodine content within the myocardium can be determined using the dedicated cardiac DECT postprocessing algorithm based on the unique X-ray absorption characteristics of iodine at different $\mathrm{kV}$ settings. The color-coded iodine distribution maps superimposed on gray-scale multiplanar reformats of the myocardium in short- and long-axis views are then generated, which can be used for the detection of myocardial ischemia [54] (Figure 1). The DE-CTP protocol commonly involves rest scan and stress testing. The rest acquisition is also used for coronary angiography evaluation. Stress testing under the administration of adenosine is subsequently performed with second bolus of iodine contrast medium [52]. The potential advantage of DECT is that myocardial perfusion defects are more conspicuous on iodine color maps compared with single-energy CT evaluation [52, 55]. Perfusion defects could be visualized by using rest-only DECTP [42]. Wang et al. [56] studied 34 patients with abnormal SPECT findings or known CAD using only rest DECT. Compared with SPECT, DECT had 68\% sensitivity and 93\% specificity for the detection of myocardial perfusion defect. Combining CCTA and rest DECT, the diagnostic accuracy was slightly improved from $86 \%$ to $88 \%$ when compared with CCTA alone using invasive coronary angiography as the reference standard. The study indicates that combination 


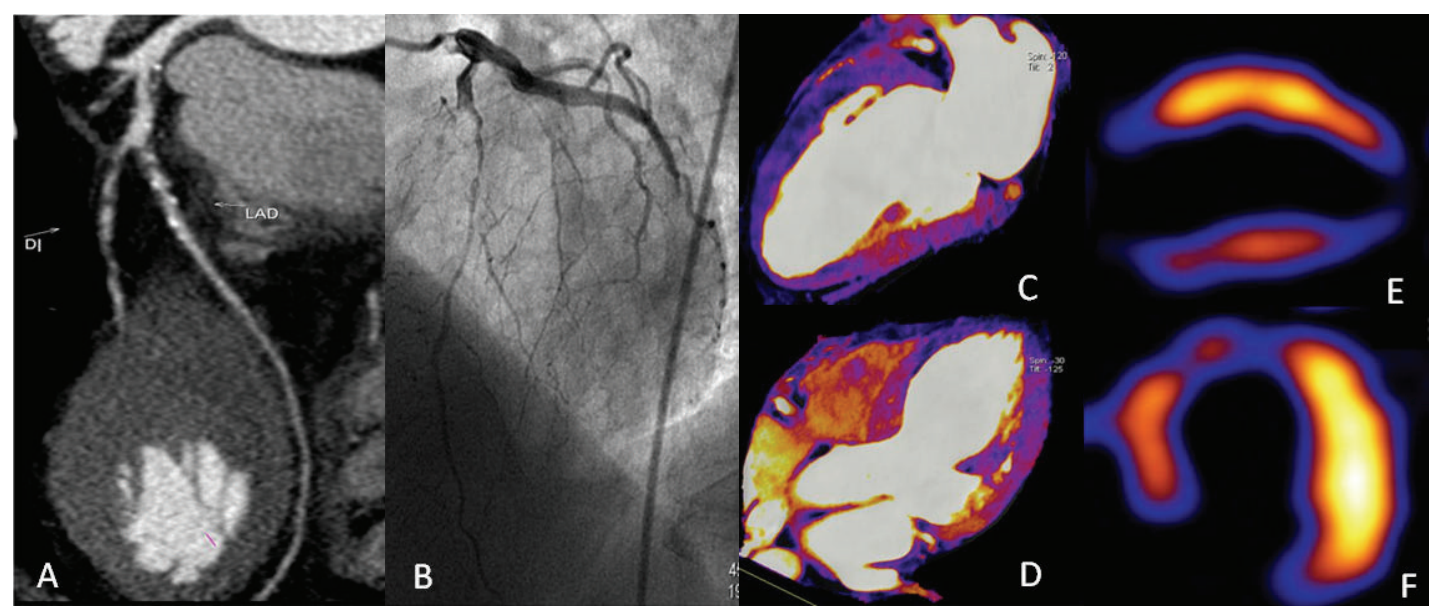

FIGURE 1: Curved multiplanar reformation of CCTA (A) shows severe stenosis in the left anterior descending artery (LAD). Coronary angiography (B) demonstrates the occlusion in the proximal LAD. DE-CTP images show hypoperfusion at the anterior, apical, septal, and inferior walls in the two and four-chamber views (C and D). SPECT-MPI images reveal perfusion defects in the same regions (E and F).

of DE-CTP and DE-CCTA may improve diagnostic performance compared to CCTA alone for significant stenosis. One study compared stress and rest DECT for the detection of myocardial perfusion defects, and the results indicate that stress DECT has superior performance for the detection of perfusion defects compared with rest DECT with CMR as the reference standard [57]. Ko et al. [58] investigated the incremental value of combined CCTA and DE-CTP for the detection of significant coronary stenoses. The study was performed by adenosine-induced stress DE-CTP and conventional coronary angiography. The sensitivity, specificity, PPV, and NPV of the CCTA alone on a per-vessel basis were $91.8 \%, 67.7 \%, 73.6 \%$, and $87.5 \%$, respectively, and these values were $93.2 \%, 85.5 \%, 88.3 \%$, and $91.4 \%$ for combining CCTA with CTP, respectively. The area under the ROC increased from 0.798 to $0.893(P=0.004)$. The results indicate that combined CCTA and CTP may provide incremental diagnostic value compared with CCTA alone for the detection of significant coronary stenoses in patients with CAD, especially in patients with heavily calcified plaques or implanted stents. To evaluate the feasibility and diagnostic accuracy of stress DECT for detecting hemodynamically significant stenosis causing reversible myocardial perfusion defect, the same group studied 41 patients with known CAD compared with stress MR perfusion and conventional coronary angiography. Stress DECT had sensitivity, specificity, and accuracy of $89 \%$, $78 \%$, and $82 \%$, respectively, for detecting segments with reversible perfusion defects compared with MR perfusion. For the detection of vascular territories with reversible perfusion defect that had hemodynamically relevant CAD, stress DECT had $89 \%$ sensitivity, $76 \%$ specificity, and $83 \%$ accuracy compared with conventional coronary angiography. The results demonstrate that stress DECT has the potential to identify stress-induced myocardial perfusion defect in patients with CAD. It should be noted that DE-CTP is more susceptible to motion artifacts during adenosine infusion and beam-hardening artifacts which mimics perfusion defects and led to false positives [59]. Recent studies also suggest that rapid $\mathrm{kV}$-switching projection-based DECT could be an effective technique for eliminating the beam-hardening effect due to the ability to reconstruct monochromatic CT images; thus, it may permit improved quantitative myocardial CTP $[60,61]$. Although the limited study results demonstrate that DECT-based evaluation of myocardial perfusion defects has the potential for the assessment of hemodynamically significant coronary stenosis in patients with CAD, more future studies will be required before DECT can be incorporated into routine clinical application.

\section{Dynamic Myocardial CT Perfusion}

Single-phase first-pass CTP is highly dependent on accurate bolus timing and thus the peak attenuation may be missed because of the acquisition of only one sample of the data. A dynamic CT perfusion scan can be performed while the scanning table is stationary or in shuttle mode $[62,63]$. During the shuttle mode imaging, data are acquired over a 30-second interval with the table moving forward and backward between both positions to cover a scan range of $73 \mathrm{~mm}$. Dynamic CTP has the advantage of capturing an entire dynamic series of contrast-enhanced myocardium at stress and at rest. From the acquired multiphase CT images, time-density curve (TDC) of the myocardium and its supply arteries are measured. The myocardial blood flow (MBF) and myocardial blood volume (MBV) can be derived from TDC using various mathematical models, leading to quantitative assessment of regional perfusion [45, 64]. Quantitative assessment of MBF offers clinical values over qualitative assessment of MBF, including the potential to detect balanced ischemia and absolute measurement of perfusion, which is better to grade the severity of ischemia [65].

The feasibility of dynamic CTP for the detection of hemodynamically significant coronary stenosis was investigated by Bamberg et al. [66] with invasive fractional flow reserve (FFR) as a reference standard. In the study, an MBF cut point of $75 \mathrm{~mL} / 100 \mathrm{~mL} / \mathrm{min}$ was determined for the differentiation between hemodynamically significant and nonsignificant coronary stenoses on the basis of maximization of the area 
under the curve. With use of estimated MBF to reclassify the lesions, the PPV was significantly increased from $49 \%$ to $78 \%$ compared with CCTA. The results suggest that dynamic CTbased stress MPI provide incremental diagnostic value for the detection of hemodynamically significant coronary artery stenosis. A recent study of stress dynamic CTP for the evaluation of myocardial ischemia and infarction was performed with CMR as the reference standard [67]. In this study, a threshold of $88 \mathrm{~mL} / 100 \mathrm{~mL} / \mathrm{min}$ for MBF was determined to indicate a perfusion defect. The diagnostic accuracy of CTP for the detection of any perfusion defect was good with $77.8 \%$ sensitivity, $75.41 \%$ specificity, $91.3 \%$ NPV, and 50.6\% PPV. Higher diagnostic accuracy was defined for transmural perfusion defects with $87.8 \%$ sensitivity and infarcted segments with $85.3 \%$ sensitivity. MBV was significantly lower in infarcted segments compared with ischemic segments. The study indicates that dynamic stress CTP provides good diagnostic accuracy for the detection of myocardial perfusion defects and has the potential to differentiate ischemic from infracted myocardium. Wang et al. [63] studied the adenosine-stress dynamic CTP compared with conventional coronary angiography and SPECT. In this study, moderate correlation was observed between adenosine-stress CTP and SPECT-MPI $(r=0.639)$. The combination of CTP with CCTA improves the diagnostic accuracy for identifying flowlimiting stenosis compared with CCTA alone.

The comparison of dynamic with single-phase CT acquisition for MPI has been recently studied. One study demonstrated similar performance of single-phase peak enhancement to the perfusion parameter MBF in the detection of ischemic myocardium [68]. Another study showed that dynamic acquisition techniques allow the identification of more subtle perfusion changes at moderate stenosis (50\%), whereas both techniques permit the identification of highgrade stenosis $(75 \%)$. The results also suggest that dynamic myocardial CTP may be more sensitive in the detection of subtle differences of myocardial perfusion compared with single-phase perfusion imaging [69]. Currently, wide detector CT system allows for covering the entire left ventricular myocardium without table movement and is more suitable for dynamic CTP imaging [62]. Manual postprocessing of dynamic myocardial perfusion $\mathrm{CT}$ data has been timeconsuming and laborious. Accordingly, the automated software for perfusion analyses is desirable to facilitate rapid interpretation and enhance reproducibility. Ebersberger et al. [70] reported the use of $3 \mathrm{D}$ semiquantitative software for the analysis of myocardial perfusion CT data with SPECT as the reference standard. The results indicate that the $3 \mathrm{D}$ semiquantitative software substantially decreases postprocessing and interpretation times while maintaining the diagnostic accuracy. Since dynamic myocardial CTP involves multiphase scans, dose issue is the major concern. Therefore, care must be taken to minimize the radiation dose while maintaining the clinically relevant information. The studies have shown promising results, and the use of dynamic myocardial CTP may allow for accurate assessment of the hemodynamically significant coronary stenosis. However, further research will be required to verify the clinical effectiveness of this approach.

\section{Delayed Enhancement CT}

Delayed enhancement CT was found to be feasible for the assessment myocardial infarction and viability. Hyperenhanced myocardial areas on delayed CT images may represent scar tissue caused by myocardial infarction. However, the contrast-to-noise ratio between the infarcted region and the normal myocardium is limited compared with CMR delayed enhancement imaging [52, 71]. One study suggested that delayed enhancement CT does not add incremental diagnostic value to CTP. Thus, delayed enhancement acquisition can be omitted to reduce radiation dose [42]. Current data are insufficient to support the use of delayed enhancement CT for the assessment of myocardial viability in patients with suspected CAD.

\section{Limitations and Prospect of CT Perfusion}

The main limitations of CTP currently are artifacts, radiation exposure, and contrast load. Artifacts can alter myocardial attenuation and interfere with myocardial perfusion analysis, which may result in misinterpretation of myocardial blood flow and perfusion defects [72]. It has been shown that in a group of asymptomatic patients with no history of CAD, who undergo CT perfusion angiography, artifacts in the posterobasal wall were identified in more than two-thirds of patients [73]. These low-attenuation pseudoperfusion defects can largely be ascribed to beam-hardening artifacts most likely from the adjacent bone structures. The high iodine concentration in the descending aorta and left ventricular chamber, however, can also result in beam-hardening artifacts that can mimic the appearance of myocardial perfusion defects and should be taken into account in the judgment of CT perfusion images. Therefore, beam-hardening artifact correction for coronary CT imaging is essential for accurate assessment of myocardial perfusion imaging. Recent study shows that image-based beam-hardening correction algorithm is feasible for the correction of beam-hardening artifacts that mimic perfusion defects. The beam-hardening correction used in the study was an image-based correction algorithm that enables individual estimation of the beam hardening from high-enhancing material and waterenhancing material for accurate calculation of the amount of beam hardening and to reconstruct a corrected image [74, 75]. The study also demonstrates that virtual monochromatic images generated using fast-switching dual-kVp technology are feasible for the correction of beam-hardening artifacts in myocardial CT imaging [71]. However, beam-hardening artifacts cannot be completely eliminated using the current beam-hardening correction algorithms [74]. In the near future, more rigorous correction algorithms for CTP imaging may improve accurate assessment of myocardial perfusion.

The increasing radiation exposure to the total population due to CT scans has raised serious concerns [76]. The main concern of exposure to ionizing radiation is the potential risk of cancer [77]. Another important limitation in myocardial CTP is the relatively high radiation dose. Stress testing requires repeated scanning that is associated with additional radiation exposure. In comparison with static CTP, dynamic 
myocardial CTP techniques involve much higher radiation exposure to the patients, because of the use of multiple phases. Notably, radiation doses are steadily decreasing as more dose-reduction strategies such as prospective ECGgating, low tube voltage, and automatic tube current modulation are performed [78-80]. In Blankstein's study [46], the average radiation exposure for the complete CT protocol including rest perfusion, stress perfusion, and delayed enhancement scan was $12.7 \pm 4.0 \mathrm{mSv}$ which is similar to SPECT $(12.7 \pm 0.4 \mathrm{mSv})$. In a recent study, by Feuchtner et al. [33], lower radiation doses can be achieved by using the latest dual-source CT with high-pitch mode, with a mean effective dose of $0.93 \pm 0.18 \mathrm{mSv}$ (range: $0.75-1.48 \mathrm{mSv}$ ) for stress and $1.59 \pm 1.3 \mathrm{mSv}$ (range: $0.53-5.8 \mathrm{mSv}$ ) for rest imaging, respectively. The use of iterative reconstruction algorithm can improve signal-to-noise ratio (SNR) and contrast-to-noise ratio (CNR) and may facilitate radiation dose savings in CTP without influencing diagnostic quality [81].

Besides the radiation, concerns about iodine contrast associated with CTP remain to be addressed, especially in patients with abnormal renal function. Combined CCTA/CTP requires doubling the iodinated contrast dose compared with CCTA alone. The repeated scanning in stress testing requires additional iodine contrast. Recent studies have demonstrated $74 \%$ reduction in radiation and $28 \%$ reduction in contrast dose without significantly compromising the ability to detect stress-induced myocardial ischemia by using lower tube voltage [78]. Therefore, optimizing the scan protocol to reduce radiation exposure and contrast dose is important for myocardial perfusion imaging. Another limitation during performing CTP is the use of beta-blockers for heart rate control which may mask ischemia [82].

Myocardial CTP has the potential to noninvasively assess both coronary artery stenosis and its functional significance at a single examination. Current evidence indicates that the combination of CCTA with CTP imaging may improve diagnostic accuracy compared with CCTA alone for the evaluation of suspected CAD. Future advances of CT technology with improved spatial and temporal resolution and detector widths, as well as beam-hardening correction algorithms, will enhance the diagnostic accuracy of CTP. CTP would be performed with very low radiation dose and free of beamhardening artifacts. More studies will be required to further define the diagnostic value of combined CCTA and CTP. Longer term follow-up studies are needed to validate the prognostic value of CTP, as well as the patient outcomes of CTP-guided clinical decision making. Although CTP has shown the potential to evaluate the hemodynamic significance of stenotic lesions, there is still no strong evidence to support the routine clinical use of this novel technique.

\section{Noninvasive FFR $_{\mathrm{CT}}$}

FFR is widely accepted as the gold standard invasive physiologic test for the assessment of lesion-specific ischemia $[83,84]$. FFR has been shown to be valuable for the identification of lesions which can benefit from revascularization or can be safely deferred. FFR-guided percutaneous coronary intervention (PCI) results in a significant reduction in major adverse events as compared to PCI-guided by angiography alone [83]. FFR is defined as the ratio of the mean coronary pressure distal to a coronary stenosis to the mean aortic pressure during maximal coronary blood flow. It can be easily measured during coronary angiography with a coronary pressure guidewire. A FFR value of 0.80 or less identifies hemodynamic significance of coronary stenosis [85]. In comparison to MPI that identifies territory-specific ischemia, the advantage of FFR is the assessment of ischemia at the lesion level. However, FFR is an invasive procedure and is not suitable for routine patient evaluation.

Recently, The FFR can be computed from standard CCTA scans $\left(\mathrm{FFR}_{\mathrm{CT}}\right)$ with the advances in computational fluid dynamics and image-based modeling. $\mathrm{FFR}_{\mathrm{CT}}$ is a novel noninvasive technique that can be used to determine the physiologic significance of coronary stenosis without any modification of CCTA protocols, additional image acquisition, or administration of medications [86]. $\mathrm{FFR}_{\mathrm{CT}}$ is calculated by computational fluid dynamic (CFD) modeling after semiautomated segmentation of coronary tree and left ventricular mass. Three-dimensional blood flow and pressure of the coronary arteries can be simulated, with blood modeled as a Newtonian fluid with incompressible Navier-Stokes equations and solved subject to appropriate initial and boundary conditions with a finite element method on parallel supercomputer $[87,88]$. The FFR ${ }_{\mathrm{CT}}$ is modeled for conditions of adenosineinduced hyperemia without adenosine infusion. This process is computationally complex and time-consuming and may require several hours. Similar to invasive FFR, FFR ${ }_{C T}$ was obtained by dividing the mean pressure distal to the coronary stenosis by the mean aortic pressure. An FFR $\mathrm{CT}<0.80$ was considered diagnostic of lesion-specific ischemia [86]. Up to now, limited $\mathrm{FFR}_{\mathrm{CT}}$ studies have been performed. In the DISCOVER-FLOW (Diagnosis of Ischemia-Causing Stenoses Obtained Via Noninvasive Fractional Flow Reserve) trial [86], FFRCT was performed on 159 vessels in 103 patients with suspected or known CAD undergoing CCTA and invasive coronary angiography. The diagnostic performance of $\mathrm{FFR}_{\mathrm{CT}}$ and CCTA stenosis for the detection of ischemia-causing lesions was assessed with invasive FFR as the reference standard. The results demonstrate that the per-vessel accuracy, sensitivity, specificity, PPV, and NPV of FFR $_{\mathrm{CT}}$ were $84.3 \%, 87.9 \%, 82.2 \%, 73.9 \%, 92.2 \%$, respectively, and were $58.5 \%, 91.4 \%, 39.6 \%, 46.5 \%, 88.9 \%$, respectively, for CCTA stenosis. The area under the ROC was 0.90 for $\mathrm{FFR}_{\mathrm{CT}}$ and 0.75 for CCTA $(P<0.001)$. The specificity and PPV were remarkably improved by FFR $_{\mathrm{CT}}$ compared with CCTA stenosis. The DeFACTO (Determination of Fractional Flow Reserve by Anatomic Computed Tomographic Angiography) trial [89], a multicenter international study which consisted of 252 patients, was designed to evaluate the performance of noninvasive $\mathrm{FFR}_{\mathrm{CT}}$ for the diagnosis of ischemia with invasive FFR as a reference standard. On a per-patient basis, diagnostic accuracy, sensitivity, specificity, PPV, and NPV of $\mathrm{FFR}_{\mathrm{CT}}$ plus CCTA were $73 \%, 90 \%, 54 \%, 67 \%$, and $84 \%$, respectively. When comparing FFR $_{\mathrm{CT}}$ with CCTA stenosis for diagnosing obstructive $\mathrm{CAD}, \mathrm{FFR}_{\mathrm{CT}}$ demonstrated improved discrimination with area under the ROC 0.81 for FFR $\mathrm{CT}_{\text {and }}$ 0.68 for CCTA $(P<0.001)$. The study results demonstrated 
improved diagnostic accuracy of $\mathrm{FFR}_{\mathrm{CT}}$ plus CCTA versus CCTA alone for the diagnosis of ischemia, although the study did not achieve its prespecified primary outcome goal of diagnostic accuracy of greater than $70 \%$ of the lower bound of the sided $95 \%$ confidence interval (95\% CI, 67\%-78\%). The study suggests the potential of $\mathrm{FFR}_{\mathrm{CT}}$ as a promising noninvasive method for the identification of individuals with ischemia. More recently, the multicenter study NXT (Analysis of Coronary Blood Flow Using CT Angiography: Next Steps) trial [90] included 251 patients scheduled to undergo clinically indicated invasive coronary angiography for suspected CAD. The diagnostic performance of $\mathrm{FFR}_{\mathrm{CT}}$ for the diagnosis of ischemia was determined with invasive FFR as the reference standard. As compared to previous studies $[86,89]$, the improved FFR $_{\mathrm{CT}}$ technology was used with an emphasis on CCTA image quality. The area under the ROC curve for $\mathrm{FFR}_{\mathrm{CT}}$ was 0.90 versus 0.81 for CCTA $(P=0.0008)$. On per-patient basis, sensitivity and specificity to identify ischemia were $86 \%$ and $79 \%$ for $\mathrm{FFR}_{\mathrm{CT}}$ versus $94 \%$ and $34 \%$ for CCTA, respectively. When compared to CCTA, the improved diagnostic performance of $\mathrm{FFR}_{\mathrm{CT}}$ is in particular with regard to specificity. The study demonstrates that $\mathrm{FFR}_{\mathrm{CT}}$ has relatively high diagnostic accuracy compared to invasive FFR for identifying hemodynamically significant CAD.

FFR $_{\mathrm{CT}}$ technology enables the "virtual stenting" modeling and may be served to predict hemodynamic effect of coronary stenting on ischemia-causing stenoses [91]. Kim et al. [92] studied the application of $\mathrm{FFR}_{\mathrm{CT}}$ in 44 patients (48 lesions) to predict FFR value changes after stenting with invasive FFR as the reference standard. Before intervention, invasive FFR was $0.70 \pm 0.14$ and increased to $0.90 \pm 0.05$ after stenting. FFR $\mathrm{CT}_{\mathrm{T}}$ was $0.70 \pm 0.15$ before intervention and increased to $0.88 \pm 0.05$ after virtual coronary stenting. There was a good correlation between FFR and FFR $\mathrm{CT}_{\text {before }}(R=$ $0.60 ; P<0.001)$ and after stenting $(R=0.55 ; P<0.001)$. This study demonstrates that virtual stenting by $\mathrm{FFR}_{\mathrm{CT}}$ is feasible and may be helpful for revascularization planning before invasive procedures.

\section{Limitations and Prospect of FFR}

There are several limitations for physiologic assessment of coronary stenosis by $\mathrm{FFR}_{\mathrm{CT}}$. First, $\mathrm{FFR}_{\mathrm{CT}}$ is calculated by computational simulation of adenosine mediated hyperemia rather than by actual administration of adenosine. Second, the value of $\mathrm{FFR}_{\mathrm{CT}}$ is influenced not only by stenosis severity but also by the presence of viable or scarred myocardium [93]. Third, the calculated $\mathrm{FFR}_{\mathrm{CT}}$ values may be lower than those of measured FFR in patients with microvascular disease, for the modeling of adenosine-induced hyperemia may overestimate the degree of vasodilation [88]. Fourth, because FFR $_{\mathrm{CT}}$ requires accurate anatomic models, numerous artifacts on CCTA may affect $\mathrm{FFR}_{\mathrm{CT}}$ calculation, including the presence of heavy calcification, motion artifacts, lower SNR, and misregistration. Therefore, CCTA data with good image quality is essential for the accuracy of FFRCT interpretation.

The results of the limited studies indicate that $\mathrm{FFR}_{\mathrm{CT}}$ is feasible for noninvasive determination of lesion-specific ischemia. Although the accuracy of $\mathrm{FFR}_{\mathrm{CT}}$ was reported as modest in several multicenter trials, this novel technique shows promise not only for diagnosing lesion-specific ischemia but also for predicting revascularization benefit. The present studies support the potential of CCTA with $\mathrm{FFR}_{\mathrm{CT}}$ as a gatekeeper to invasive coronary angiography and revascularization. Further improvements in the technology or new algorithms for a reliable estimation of FFR will be a prerequisite for wide acceptance of $\mathrm{FFR}_{\mathrm{CT}}$, and further studies are needed to determine its clinical utility compared with other noninvasive testing. Combination of CCTA and $\mathrm{FFR}_{\mathrm{CT}}$ might permit evaluation of CAD in a "one-stop-shop" approach in the future.

\section{Transluminal Attenuation Gradient (TAG)}

Assessment of the change in intraluminal contrast attenuation across a coronary stenosis on CCTA may allow prediction of functional significance of coronary stenosis [94]. A methodology called "coronary opacification" (CO) difference, defined as the contrast attenuation difference across a stenosis, has been proposed for functional prediction of stenosis. In the study, by Chow et al. [95], attenuation values of coronary lumen were measured before and after stenoses and normalized to the descending aorta on the same axial slice, because the image acquisition is not temporally uniform using 64-slice CT. Corrected coronary opacification (CCO) differences were compared with the severity of coronary stenosis and thrombolysis in myocardial infarction (TIMI) flow at invasive coronary angiography. The results indicate that changes in $\mathrm{CCO}$ across coronary stenoses seem to predict abnormal resting coronary blood flow.

However, the measurement of gradients across coronary stenoses is inherently more robust than evaluation of opacification difference. Transluminal attenuation gradient (TAG) is defined as the linear regression coefficient between luminal attenuation $(\mathrm{Hu})$ and axial distance along the vessel from the coronary ostium. TAG is reflected by the kinetics of the iodinated contrast media within the coronary arteries. Study confirmed that opacification gradients exist in patients with normal coronary arteries. The TAG along the course of a coronary artery can be reproducibly evaluated with CCTA [96]. The hypothesis of TAG as a functional test is that contrast attenuation should fall off more rapidly across a lesion with functionally significant stenosis [97]. Recent studies suggest that TAG may provide useful information for the assessment of functional significance of coronary stenosis with the comparison of invasive FFR. TAG significantly improves both sensitivity and specificity over CCTA stenosis degree alone [94].

It has been reported that TAG has the potential to improve the classification of stenosis severity on 64-slice CCTA compared with coronary angiography as a reference standard, especially in calcified lesions, which is particularly useful because CCTA is limited in accuracy when lesions are calcified [98]. The limitation of TAG analysis, using 64-slice CCTA, pointed out by the authors, is that attenuation along a vessel does not reflect contrast density at a single time point, potentially limiting the diagnostic performance of the TAG method. The use of 320-detector row CT with whole-heart 
volume to be imaged in a single gantry rotation enables noninvasive quantitative assessment of coronary contrast changes with temporal uniformity, which may be ideal for TAG functional assessment of a coronary arterial stenosis $[94,96]$. Comparing labor-intensive manual TAG calculation methods, latest automated gradient software package can reduce computation time with high accuracy and reproducibility [99].

Combined TAG and CCTA assessment may have incremental value over CCTA alone for detecting functionally significant coronary stenoses, especially in severely calcified lesions. TAG depends on the luminal attenuation values, and the accuracy of quantification may be influenced by various artifacts on CCTA. So, maintaining image quality of CCTA is important for TAG analysis. TAG assessment has particular attraction, as it does not require additional scan or complex computation compared with CT perfusion or $\mathrm{FFR}_{\mathrm{CT}}$. However, given the limited evidence so far, larger studies with current acquisition and reconstruction protocols as well as new analysis software are required to validate the diagnostic and prognostic value of this approach.

\section{Summary}

CCTA has become an important noninvasive imaging modality in the diagnosis of CAD. CCTA enables accurate evaluation of coronary artery stenosis. However, CCTA provides limited information on the physiological significance of stenotic lesions. There is a great interest in evaluating both coronary anatomy and its hemodynamic significance during a single examination. Combination of anatomic and physiologic information would be beneficial for clinical decision making, particularly in lesions with moderate stenoses. Recently, novel CT techniques which can provide both anatomical and functional assessments of CAD were developed, including myocardial CTP and noninvasive $\mathrm{FFR}_{\mathrm{CT}}$, which simulate FFR from CT data using CFD method and TAG derived from the gradient in contrast opacification along a coronary artery. The current studies have demonstrated that these methods are feasible for noninvasive assessment of CAD and have the potential to provide incremental value in detecting functionally significant coronary stenosis over CCTA alone. Although the currently available data are promising, these approaches are still in its early stage, and their diagnostic values still need further validation. Further research is required to identify the prognostic value and clinical outcomes of decision making based on these techniques.

\section{Conflict of Interests}

The authors declare that there is no conflict of interests regarding the publication of this paper.

\section{Acknowledgments}

The authors thank Dr. Rui Wang at Beijing Anzhen Hospital, Capital Medical University, for providing the figures. This work has been supported by a Grant for Excellent Talents from Beijing city (2011D003034000030), Natural Science Foundation of China (Grant 81271542), and a Grant for High Levels of Health Technical Personnel in Beijing City Health System (2013-3-011).

\section{References}

[1] D. B. Mark, D. S. Berman, M. J. Budoff et al., "CCF/ACR/AHA/ NASCI/SAIP/SCAI/SCCT 2010 expert consensus document on coronary computed tomographic angiography: a report of the American College of Cardiology Foundation Task Force on Expert Consensus Documents," Circulation, vol. 121, no. 22, pp. 2509-2543, 2010.

[2] J. M. Miller, C. E. Rochitte, M. Dewey et al., "Diagnostic performance of coronary angiography by 64-row CT," The New England Journal of Medicine, vol. 359, no. 22, pp. 2324-2336, 2008.

[3] M. J. Budoff, D. Dowe, J. G. Jollis et al., "Diagnostic performance of 64-multidetector row coronary computed tomographic angiography for evaluation of coronary artery stenosis in individuals without known coronary artery disease: results from the prospective multicenter ACCURACY (Assessment by Coronary Computed Tomographic Angiography of Individuals Undergoing Invasive Coronary Angiography) trial," Journal of the American College of Cardiology, vol. 52, no. 21, pp. 1724-1732, 2008.

[4] P. Schlattmann, G. M. Schuetz, and M. Dewey, "Influence of coronary artery disease prevalence on predictive values of coronary CT angiography: a meta-regression analysis," European Radiology, vol. 21, no. 9, pp. 1904-1913, 2011.

[5] P. K. Vanhoenacker, M. H. Heijenbrok-Kal, R. van Heste et al., "Diagnostic performance of multidetector CT angiography for assessment of coronary artery disease: meta-analysis," Radiology, vol. 244, no. 2, pp. 419-428, 2007.

[6] L. J. Shaw, J. Hausleiter, S. Achenbach et al., "Coronary computed tomographic angiography as a gatekeeper to invasive diagnostic and surgical procedures: Results from the multicenter confirm (coronary ct angiography evaluation for clinical outcomes: an international multicenter) registry," Journal of the American College of Cardiology, vol. 60, no. 20, pp. 2103-2114, 2012.

[7] S. Voros, S. Rinehart, Z. Qian et al., "Coronary atherosclerosis imaging by coronary CT angiography: current status, correlation with intravascular interrogation and meta-analysis," JACC: Cardiovascular Imaging, vol. 4, no. 5, pp. 537-548, 2011.

[8] U. Hoffmann, F. Moselewski, K. Nieman et al., "Noninvasive assessment of plaque morphology and composition in culprit and stable lesions in acute coronary syndrome and stable lesions in stable angina by multidetector computed tomography," Journal of the American College of Cardiology, vol. 47, no. 8, pp. 16551662, 2006.

[9] M. Otsuka, N. Bruining, N. C. van Pelt et al., "Quantification of coronary plaque by 64 -slice computed tomography: a comparison with quantitative intracoronary ultrasound," Investigative Radiology, vol. 43, no. 5, pp. 314-321, 2008.

[10] S. Motoyama, M. Sarai, H. Harigaya et al., "Computed tomographic angiography characteristics of atherosclerotic plaques subsequently resulting in acute coronary syndrome," Journal of the American College of Cardiology, vol. 54, no. 1, pp. 49-57, 2009.

[11] J. Abdulla, C. Asferg, and K. F. Kofoed, "Prognostic value of absence or presence of coronary artery disease determined 
by 64-slice computed tomography coronary angiography A systematic review and meta-analysis," The International Journal of Cardiovascular Imaging, vol. 27, no. 3, pp. 413-420, 2011.

[12] J. M. van Werkhoven, O. Gaemperli, J. D. Schuijf et al., "Multislice computed tomography coronary angiography for risk stratification in patients with an intermediate pretest likelihood," Heart, vol. 95, no. 19, pp. 1607-1611, 2009.

[13] J. K. Min, A. Dunning, F. Y. Lin et al., "Age- and sex-related differences in all-cause mortality risk based on coronary computed tomography angiography findings: Results from the international multicenter CONFIRM (Coronary CT Angiography Evaluation for Clinical Outcomes: An International Multicenter Registry) of 23,854 patients without known coronary artery disease," Journal of the American College of Cardiology, vol. 58, no. 8, pp. 849-860, 2011.

[14] L. J. Shaw and J. Narula, "Coronary CT angiography: an established, not emerging, basis of diagnosis and risk stratification," JACC: Cardiovascular Imaging, vol. 4, no. 5, pp. 565-566, 2011.

[15] A. Arbab-Zadeh, J. M. Miller, C. E. Rochitte et al., "Diagnostic accuracy of computed tomography coronary angiography according to pre-test probability of coronary artery disease and severity of coronary arterial calcification. The CORE-64 (Coronary Artery Evaluation Using 64-Row Multidetector Computed Tomography Angiography) International Multicenter Study," Journal of the American College of Cardiology, vol. 59, no. 4, pp. 379-387, 2012.

[16] S. E. Nissen, "Coronary computed tomography angiography: the challenge of coronary calcium," Journal of the American College of Cardiology, vol. 59, no. 4, pp. 388-389, 2012.

[17] J. K. Min, L. J. Shaw, and D. S. Berman, "The present state of coronary computed tomography angiography a process in evolution," Journal of the American College of Cardiology, vol. 55, no. 10, pp. 957-965, 2010.

[18] G. A. Beller and B. L. Zaret, "Contributions of nuclear cardiology to diagnosis and prognosis of patients with coronary artery disease," Circulation, vol. 101, no. 12, pp. 1465-1478, 2000.

[19] L. J. Shaw, D. S. Berman, D. J. Maron et al., "Optimal medical therapy with or without percutaneous coronary intervention to reduce ischemic burden: results from the Clinical Outcomes Utilizing Revascularization and Aggressive Drug Evaluation (COURAGE) trial nuclear substudy," Circulation, vol. 117, no. 10, pp. 1283-1291, 2008.

[20] N. H. J. Pijls, B. De Bruyne, K. Peels et al., "Measurement of fractional flow reserve to assess the functional severity of coronaryartery stenoses," The New England Journal of Medicine, vol. 334, no. 26, pp. 1703-1708, 1996.

[21] P. A. L. Tonino, W. F. Fearon, B. de Bruyne et al., "Angiographic versus functional severity of coronary artery stenoses in the FAME study fractional flow reserve versus angiography in multivessel evaluation," Journal of the American College of Cardiology, vol. 55, no. 25, pp. 2816-2821, 2010.

[22] S. Rispler, Z. Keidar, E. Ghersin et al., "Integrated single-photon emission computed tomography and computed tomography coronary angiography for the assessment of hemodynamically significant coronary artery lesions," Journal of the American College of Cardiology, vol. 49, no. 10, pp. 1059-1067, 2007.

[23] J. D. Schuijf, W. Wijns, J. W. Jukema et al., "Relationship between noninvasive coronary angiography with multi-slice computed tomography and myocardial perfusion imaging," Journal of the American College of Cardiology, vol. 48, no. 12, pp. 2508-2514, 2006.
[24] M. F. Di Carli, S. Dorbala, Z. Curillova et al., "Relationship between CT coronary angiography and stress perfusion imaging in patients with suspected ischemic heart disease assessed by integrated PET-CT imaging," Journal of Nuclear Cardiology, vol. 14, no. 6, pp. 799-809, 2007.

[25] A. Notghi and C. S. Low, "Myocardial perfusion scintigraphy: past, present and future," British Journal of Radiology, vol. 84, no. 3, pp. S229-S236, 2011.

[26] S. R. Underwood, C. Anagnostopoulos, M. Cerqueira et al., "Myocardial perfusion scintigraphy: the evidence," European Journal of Nuclear Medicine and Molecular Imaging, vol. 31, no. 2, pp. 261-291, 2004.

[27] T. Zhou, L.-F. Yang, J.-L. Zhai et al., "SPECT myocardial perfusion versus fractional flow reserve for evaluation of functional ischemia: a meta analysis," European Journal of Radiology, vol. 83, no. 6, pp. 951-956, 2014.

[28] R. A. Dvorak, R. K. J. Brown, and J. R. Corbett, "Interpretation of SPECT/CT myocardial perfusion images: common artifacts and quality control techniques," Radiographics, vol. 31, no. 7, pp. 2041-2057, 2011.

[29] W. H. Aarnoudse, K.-J. B. M. Botman, and N. H. J. Pijls, "False-negative myocardial scintigraphy in balanced threevessel disease, revealed by coronary pressure measurement," International Journal of Cardiovascular Interventions, vol. 5, no. 2, pp. 67-71, 2003.

[30] R. Nakazato, D. S. Berman, E. Alexanderson, and P. Slomka, "Myocardial perfusion imaging with PET," Imaging in Medicine, vol. 5, no. 1, pp. 35-46, 2013.

[31] T. M. Bateman, G. V. Heller, A. I. McGhie et al., "Diagnostic accuracy of rest/stress ECG-gated Rb-82 myocardial perfusion PET: comparison with ECG-gated Tc-99m sestamibi SPECT," Journal of Nuclear Cardiology, vol. 13, no. 1, pp. 24-33, 2006.

[32] K. R. Nandalur, B. A. Dwamena, A. F. Choudhri, S. R. Nandalur, P. Reddy, and R. C. Carlos, "Diagnostic performance of positron emission tomography in the detection of coronary artery disease: a meta-analysis," Academic Radiology, vol. 15, no. 4, pp. 444-451, 2008.

[33] G. Feuchtner, R. Goetti, A. Plass et al., "Adenosine stress high-pitch 128-slice dual-source myocardial computed tomography perfusion for imaging of reversible myocardial ischemia comparison with magnetic resonance imaging," Circulation: Cardiovascular Imaging, vol. 4, no. 5, pp. 540-549, 2011.

[34] K. R. Nandalur, B. A. Dwamena, A. F. Choudhri, M. R. Nandalur, and R. C. Carlos, "Diagnostic performance of stress cardiac magnetic resonance imaging in the detection of coronary artery disease: a meta-analysis," Journal of the American College of Cardiology, vol. 50, no. 14, pp. 1343-1353, 2007.

[35] K. Hosokawa, A. Kurata, T. Kido et al., "Transmural perfusion gradient in adenosine triphosphate stress myocardial perfusion computed tomography," Circulation Journal, vol. 75, no. 8, pp. 1905-1912, 2011.

[36] K. Iwasaki and T. Matsumoto, "Myocardial perfusion defect in patients with coronary artery disease demonstrated by $64-$ multidetector computed tomography at rest," Clinical Cardiology, vol. 34, no. 7, pp. 454-460, 2011.

[37] A. Rossi, D. Merkus, E. Klotz, N. Mollet, P. J. de Feyter, and G. P. Krestin, "Stress myocardial perfusion imaging with multidetector CT," Radiology, vol. 270, no. 1, pp. 25-46, 2014.

[38] J. L. Busch, A. M. Alessio, J. H. Caldwell et al., "Myocardial hypo-enhancement on resting computed tomography angiography images accurately identifies myocardial hypoperfusion," 
Journal of Cardiovascular Computed Tomography, vol. 5, no. 6, pp. 412-420, 2011.

[39] J. M. Troupis, A. Karge, S. Seneviratne et al., "Myocardial density analysis utilizing automated myocardial defect analysis software on resting 320-detector MDCT," The International Journal of Cardiovascular Imaging, vol. 29, no. 5, pp. 1121-1127, 2013.

[40] B. B. Ghoshhajra, P. Maurovich-Horvat, T. Techasith et al., "Infarct detection with a comprehensive cardiac ct protocol," Journal of Cardiovascular Computed Tomography, vol. 6, no. 1, pp. 14-23, 2012.

[41] M. Nagao, H. Matsuoka, H. Kawakami et al., "Myocardial ischemia in acute coronary syndrome: assessment using 64MDCT,' The American Journal of Roentgenology, vol. 193, no. 4, pp. 1097-1106, 2009.

[42] F. G. Meinel, C. N. de Cecco, U. J. Schoepf et al., "Firstarterial-pass dual-energy CT for assessment of myocardial blood supply: do we need rest, stress, and delayed acquisition? Comparison with SPECT," Radiology, vol. 270, no. 3, pp. 708716, 2014.

[43] T. Techasith and R. C. Cury, "Stress myocardial ct perfusion: an update and future perspective," JACC: Cardiovascular Imaging, vol. 4, no. 8, pp. 905-916, 2011.

[44] R. W. Nesto and G. J. Kowalchuk, "The ischemic cascade: temporal sequence of hemodynamic, electrocardiographic and symptomatic expressions of ischemia," The American Journal of Cardiology, vol. 59, no. 7, pp. 23C-30C, 1987.

[45] B. S. Ko, J. D. Cameron, T. DeFrance, and S. K. Seneviratne, "CT stress myocardial perfusion imaging using Multidetector CTa review," Journal of Cardiovascular Computed Tomography, vol. 5, no. 6, pp. 345-356, 2011.

[46] R. Blankstein, L. D. Shturman, I. S. Rogers et al., "Adenosineinduced stress myocardial perfusion imaging using dual-source cardiac computed tomography," Journal of the American College of Cardiology, vol. 54, no. 12, pp. 1072-1084, 2009.

[47] B. S. Ko, J. D. Cameron, I. T. Meredith et al., "Computed tomography stress myocardial perfusion imaging in patients considered for revascularization: a comparison with fractional flow reserve," European Heart Journal, vol. 33, no. 1, pp. 67-77, 2012.

[48] C. E. Rochitte, R. T. George, M. Y. Chen et al., "Computed tomography angiography and perfusion to assess coronary artery stenosis causing perfusion defects by single photon emission computed tomography: the CORE320 study," European Heart Journal, vol. 35, no. 17, pp. 1120-1130, 2014.

[49] R. T. George, A. Arbab-Zadeh, R. J. Cerci et al., "Diagnostic performance of combined noninvasive coronary angiography and myocardial perfusion imaging using 320-MDCT: the CT angiography and perfusion methods of the CORE320 multicenter multinational diagnostic study," American Journal of Roentgenology, vol. 197, no. 4, pp. 829-837, 2011.

[50] M. Rief, E. Zimmermann, F. Stenzel et al., "Computed tomography angiography and myocardial computed tomography perfusion in patients with coronary stents: prospective intraindividual comparison with conventional coronary angiography," Journal of the American College of Cardiology, vol. 62, no. 16, pp. 1476-1485, 2013.

[51] T. R. C. Johnson, B. Krauß, M. Sedlmair et al., "Material differentiation by dual energy CT: initial experience," European Radiology, vol. 17, no. 6, pp. 1510-1517, 2007.

[52] R. Vliegenthart, G. J. Pelgrim, U. Ebersberger, G. W. Rowe, M. Oudkerk, and U. J. Schoepf, "Dual-energy CT of the heart," The
American Journal of Roentgenology, vol. 199, supplement 5, pp. S54-63, 2012.

[53] A. So, J. Hsieh, S. Narayanan et al., "Dual-energy CT and its potential use for quantitative myocardial CT perfusion," Journal of Cardiovascular Computed Tomography, vol. 6, no. 5, pp. 308317, 2012.

[54] B. Ruzsics, H. Lee, P. L. Zwerner, M. Gebregziabher, P. Costello, and U. J. Schoepf, "Dual-energy CT of the heart for diagnosing coronary artery stenosis and myocardial ischemia-initial experience," European Radiology, vol. 18, no. 11, pp. 2414-2424, 2008.

[55] E. Arnoldi, Y. S. Lee, B. Ruzsics et al., "CT detection of myocardial blood volume deficits: dual-energy CT compared with single-energy CT spectra," Journal of Cardiovascular Computed Tomography, vol. 5, no. 6, pp. 421-429, 2011.

[56] R. Wang, W. Yu, Y. Wang et al., "Incremental value of dualenergy CT to coronary CT angiography for the detection of significant coronary stenosis: comparison with quantitative coronary angiography and single photon emission computed tomography," International Journal of Cardiovascular Imaging, vol. 27, no. 5, pp. 647-656, 2011.

[57] S. M. Ko, J. H. Park, H. K. Hwang, and M. G. Song, "Direct comparison of stress- and rest-dual-energy computed tomography for detection of myocardial perfusion defect," The International Journal of Cardiovascular Imaging, vol. 30, supplement 1, pp. 4153, 2014.

[58] S. M. Ko, J. W. Choi, H. K. Hwang, M. G. Song, J. K. Shin, and H. K. Chee, "Diagnostic performance of combined noninvasive anatomic and functional assessment with dualsource CT and adenosine- induced stress dual-energy CT for detection of significant coronary stenosis," American Journal of Roentgenology, vol. 198, no. 3, pp. 512-520, 2012.

[59] S. M. Ko, J. W. Choi, M. G. Song et al., "Myocardial perfusion imaging using adenosine-induced stress dual-energy computed tomography of the heart: comparison with cardiac magnetic resonance imaging and conventional coronary angiography," European Radiology, vol. 21, no. 1, pp. 26-35, 2011.

[60] A. So, J. Hsieh, Y. Imai et al., "Prospectively ECG-triggered rapid $\mathrm{kV}$-switching dual-energy $\mathrm{CT}$ for quantitative imaging of myocardial perfusion," JACC: Cardiovascular Imaging, vol. 5, no. 8, pp. 829-836, 2012.

[61] A. So, T. Y. Lee, Y. Imai et al., "Quantitative myocardial perfusion imaging using rapid $\mathrm{kVp}$ switch dual-energy $\mathrm{CT}$ : preliminary experience," Journal of Cardiovascular Computed Tomography, vol. 5, no. 6, pp. 430-442, 2011.

[62] D. Muenzel, S. Kabus, B. Gramer et al., "Dynamic CT perfusion imaging of the myocardium: a technical note on improvement of image quality," PLoS ONE, vol. 8, no. 10, Article ID e75263, 2013.

[63] Y. Wang, L. Qin, X. Shi et al., "Adenosine-stress dynamic myocardial perfusion imaging with second-generation dualsource CT: Comparison with conventional catheter coronary angiography and SPECT nuclear myocardial perfusion imaging," The American Journal of Roentgenology, vol. 198, no. 3, pp. 521-529, 2012.

[64] F. Bamberg, E. Klotz, T. Flohr et al., "Dynamic myocardial stress perfusion imaging using fast dual-source CT with alternating table positions: initial experience," European Radiology, vol. 20, no. 5, pp. 1168-1173, 2010.

[65] M. Bindschadler, D. Modgil, K. R. Branch, P. J. La Riviere, and A. M. Alessio, "Comparison of blood flow models and acquisitions for quantitative myocardial perfusion estimation from dynamic 
CT," Physics in Medicine and Biology, vol. 59, no. 7, pp. 1533-1556, 2014.

[66] F. Bamberg, A. Becker, F. Schwarz et al., "Detection of hemodynamically significant coronary artery stenosis: incremental diagnostic value of dynamic CT-based myocardial perfusion imaging," Radiology, vol. 260, no. 3, pp. 689-698, 2011.

[67] F. Bamberg, R. P. Marcus, A. Becker et al., "Dynamic myocardial CT perfusion imaging for evaluation of myocardial ischemia as determined by MR imaging," JACC: Cardiovascular Imaging, vol. 7, no. 3, pp. 267-277, 2014.

[68] A. M. Huber, V. Leber, B. M. Gramer et al., "Myocardium: dynamic versus single-shot CT perfusion imaging," Radiology, vol. 269, no. 2, pp. 378-386, 2013.

[69] F. Schwarz, R. Hinkel, E. Baloch et al., "Myocardial CT perfusion imaging in a large animal model: comparison of dynamic versus single-phase acquisitions," JACC: Cardiovascular Imaging, vol. 6, no. 12, pp. 1229-1238, 2013.

[70] U. Ebersberger, R. P. Marcus, U. J. Schoepf et al., "Dynamic CT myocardial perfusion imaging: performance of $3 \mathrm{D}$ semiautomated evaluation software," European Radiology, vol. 24, no. 1, pp. 191-199, 2014.

[71] B. L. Gerber, B. Belge, G. J. Legros et al., "Characterization of acute and chronic myocardial infarcts by multidetector computed tomography: comparison with contrast-enhanced magnetic resonance," Circulation, vol. 113, no. 6, pp. 823-833, 2006.

[72] E. E. Van Der Wall, J. D. Schuijf, J. J. Bax, J. W. Jukema, and M. J. Schalij, "CT perfusion angiography; Beware of artifacts!" The International Journal of Cardiovascular Imaging, vol. 26, no. 3, pp. 355-358, 2010.

[73] G. A. Rodríguez-Granillo, M. A. Rosales, E. Degrossi, and A. E. Rodriguez, "Signal density of left ventricular myocardial segments and impact of beam hardening artifact: implications for myocardial perfusion assessment by multidetector CT coronary angiography," The International Journal of Cardiovascular Imaging, vol. 26, no. 3, pp. 345-354, 2010.

[74] K. Kitagawa, R. T. George, A. Arbab-Zadeh, J. A. C. Lima, and A. C. Lardo, "Characterization and correction of beam-hardening artifacts during dynamic volume CT assessment of myocardial perfusion," Radiology, vol. 256, no. 1, pp. 111-118, 2010.

[75] M. Yamada, M. Jinzaki, S. Kuribayashi, N. Imanishi, K. Funato, and S. Aiso, "Beam-hardening correction for virtual monochromatic imaging of myocardial perfusion via fast-switching dual$\mathrm{kVp}$ 64-slice computed tomography: a pilot study using a human heart specimen," Circulation Journal, vol. 76, no. 7, pp. 1799-1801, 2012.

[76] D. J. Brenner and E. J. Hall, "Computed tomography-an increasing source of radiation exposure," The New England Journal of Medicine, vol. 357, no. 22, pp. 2277-2284, 2007.

[77] A. J. Einstein, M. J. Henzlova, and S. Rajagopalan, "Estimating risk of cancer associated with radiation exposure from 64-slice computed tomography coronary angiography," Journal of the American Medical Association, vol. 298, no. 3, pp. 317-323, 2007.

[78] A. R. Patel, J. A. Lodato, S. Chandra et al., "Detection of myocardial perfusion abnormalities using ultra-low radiation dose regadenoson stress multidetector computed tomography," Journal of Cardiovascular Computed Tomography, vol. 5, no. 4, pp. 247-254, 2011.

[79] M. Fujita, K. Kitagawa, T. Ito et al., "Dose reduction in dynamic CT stress myocardial perfusion imaging: comparison of 80-kV/370-mAs and 100-kV/300-mAs protocols," European Radiology, vol. 24, no. 3, pp. 748-755, 2014.
[80] S. M. Kim, Y. N. Kim, and Y. H. Choe, "Adenosine-stress dynamic myocardial perfusion imaging using 128-slice dualsource CT: optimization of the CT protocol to reduce the radiation dose," The International Journal of Cardiovascular Imaging, vol. 29, no. 4, pp. 875-884, 2013.

[81] B. M. Gramer, D. Muenzel, V. Leber et al., "Impact of iterative reconstruction on CNR and SNR in dynamic myocardial perfusion imaging in an animal model," European Radiology, vol. 22, no. 12, pp. 2654-2661, 2012.

[82] G. J. Zoghbi, T. A. Dorfman, and A. E. Iskandrian, "The effects of medications on myocardial perfusion," Journal of the American College of Cardiology, vol. 52, no. 6, pp. 401-416, 2008.

[83] P. A. L. Tonino, B. De Bruyne, N. H. J. Pijls et al., "Fractional flow reserve versus angiography for guiding percutaneous coronary intervention," The New England Journal of Medicine, vol. 360, no. 3, pp. 213-224, 2009.

[84] B. De Bruyne, N. H. J. Pijls, B. Kalesan et al., "Fractional flow reserve-guided PCI versus medical therapy in stable coronary disease," The New England Journal of Medicine, vol. 367, no. 11, pp. 991-1001, 2012.

[85] N. H. J. Pijls and B. De Bruyne, "Coronary pressure measurement and fractional flow reserve," Heart, vol. 80, no. 6, pp. 539542, 1998.

[86] B.-K. Koo, A. Erglis, J.-H. Doh et al., "Diagnosis of ischemiacausing coronary stenoses by noninvasive fractional flow reserve computed from coronary computed tomographic angiograms: results from the prospective multicenter DISCOVER-FLOW (Diagnosis of Ischemia-Causing Stenoses Obtained Via Noninvasive Fractional Flow Reserve) study," Journal of the American College of Cardiology, vol. 58, no. 19, pp. 1989-1997, 2011.

[87] H. J. Kim, I. E. Vignon-Clementel, J. S. Coogan, C. A. Figueroa, K. E. Jansen, and C. A. Taylor, "Patient-specific modeling of blood flow and pressure in human coronary arteries," Annals of Biomedical Engineering, vol. 38, no. 10, pp. 3195-3209, 2010.

[88] C. A. Taylor, T. A. Fonte, and J. K. Min, "Computational fluid dynamics applied to cardiac computed tomography for noninvasive quantification of fractional flow reserve: scientific basis," Journal of the American College of Cardiology, vol. 61, no. 22, pp. 2233-2241, 2013.

[89] J. K. Min, J. Leipsic, M. J. Pencina et al., "Diagnostic accuracy of fractional flow reserve from anatomic CT angiography," Journal of the American Medical Association, vol. 308, no. 12, pp. 12371245, 2012.

[90] B. L. Nørgaard, J. Leipsic, S. Gaur et al., "Diagnostic performance of noninvasive fractional flow reserve derived from coronary computed tomography angiography in suspected coronary artery disease: the NXT trial (Analysis of Coronary Blood Flow Using CT Angiography: Next Steps)," Journal of the American College of Cardiology, vol. 63, no. 12, pp. 1145-1155, 2014.

[91] T. A. Magalhães and J. A. C. Lima, "Defining the flowlimiting stenosis noninvasively for management of patients with coronary artery disease," JACC: Cardiovascular Interventions, vol. 7, no. 1, pp. 79-80, 2014.

[92] K. H. Kim, J. H. Doh, B. K. Koo et al., "A novel noninvasive technology for treatment planning using virtual coronary stenting and computed tomography-derived computed fractional flow reserve," JACC: Cardiovascular Interventions, vol. 7, no. 1, pp. 7278, 2014.

[93] A. R. De Caterina, A. M. Leone, and F. Crea, "Limitations of noninvasive measurement of fractional flow reserve from 
coronary computed tomography angiography," Journal of the American College of Cardiology, vol. 59, no. 15, pp. 1408-1410, 2012.

[94] D. T. L. Wong, B. S. Ko, J. D. Cameron et al., "Transluminal attenuation gradient in coronary computed tomography angiography is a novel noninvasive approach to the identification of functionally significant coronary artery stenosis: a comparison with fractional flow reserve," Journal of the American College of Cardiology, vol. 61, no. 12, pp. 1271-1279, 2013.

[95] B. J. W. Chow, M. Kass, O. Gagn et al., "Can differences in corrected coronary opacification measured with computed tomography predict resting coronary artery flow?" Journal of the American College of Cardiology, vol. 57, no. 11, pp. 1280-1288, 2011.

[96] M. L. Steigner, D. Mitsouras, A. G. Whitmore et al., "Iodinated contrast opacification gradients in normal coronary arteries imaged with prospectively ECG-gated single heart beat 320detector row computed tomography," Circulation: Cardiovascular Imaging, vol. 3, no. 2, pp. 179-186, 2010.

[97] A. J. Einstein, "TAG-is it it? Improving coronary computed tomography angiography with the isotemporal transluminal contrast attenuation gradient," Journal of the American College of Cardiology, vol. 61, no. 12, pp. 1280-1282, 2013.

[98] J. H. Choi, J. K. Min, T. M. Labounty et al., "Intracoronary transluminal attenuation gradient in coronary CT angiography for determining coronary artery stenosis," JACC Cardiovascular Imaging, vol. 4, no. 11, pp. 1149-1157, 2011.

[99] Y. S. Chatzizisis, E. George, T. Cai et al., "Accuracy and reproducibility of automated, standardized coronary transluminal attenuation gradient measurements," The International Journal of Cardiovascular Imaging, vol. 30, no. 6, pp. 1181-1189, 2014. 


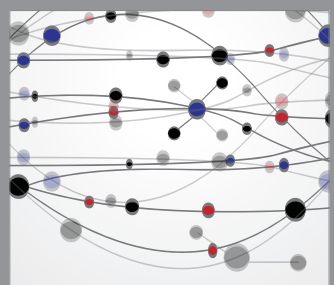

The Scientific World Journal
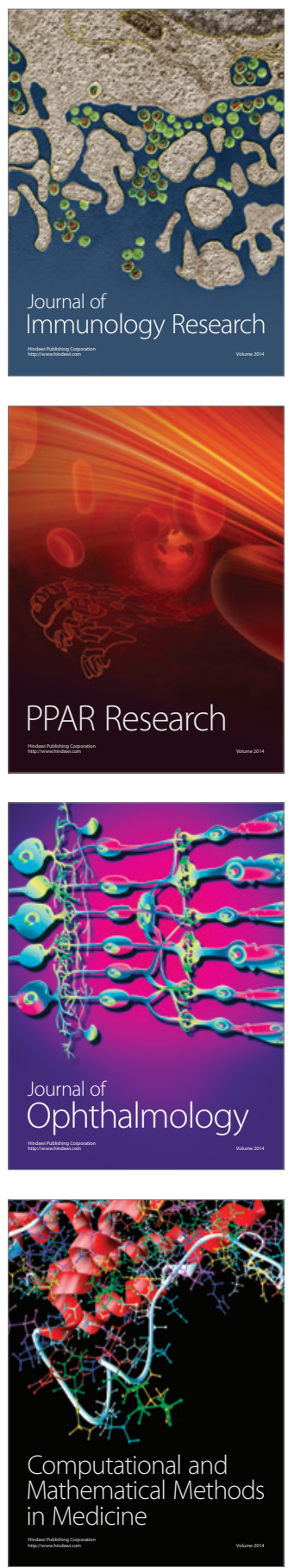

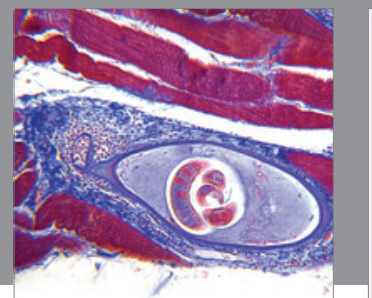

Gastroenterology

Research and Practice
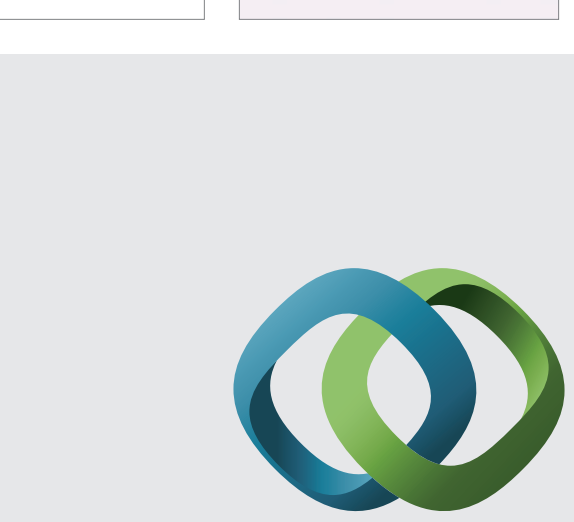

\section{Hindawi}

Submit your manuscripts at

http://www.hindawi.com
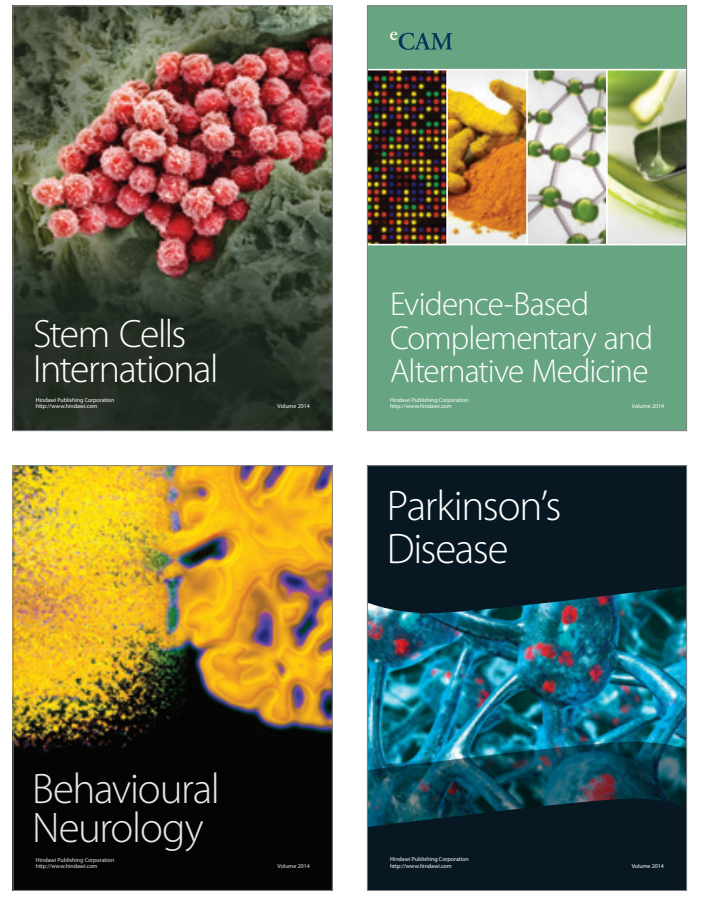
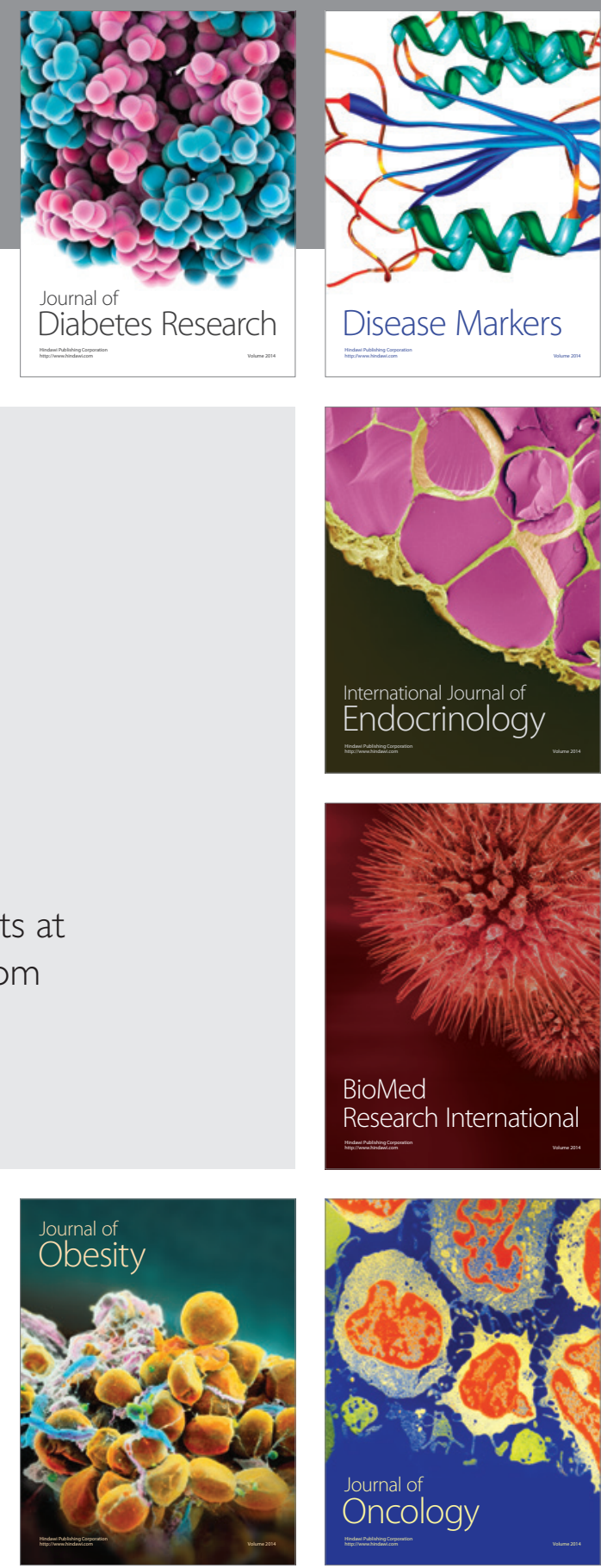

Disease Markers
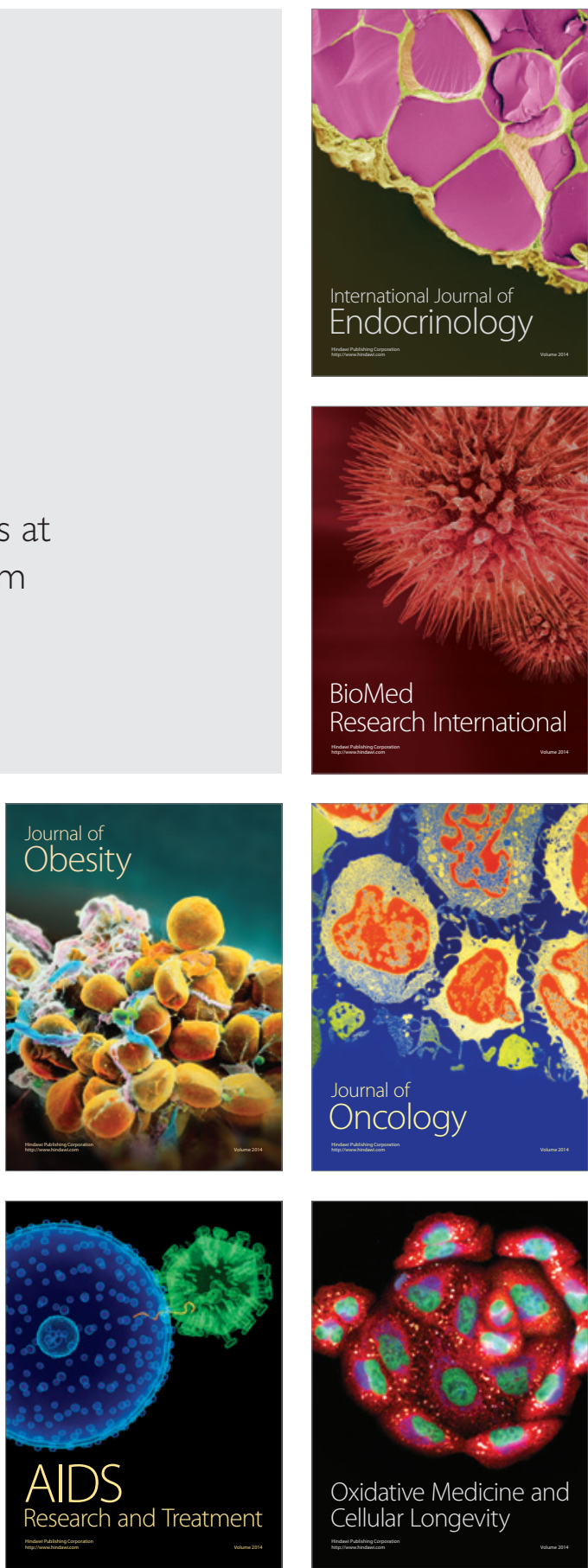